\title{
Low Cross-Polarization Slab Waveguide Filter for Narrow-Wall Slotted Waveguide Array Antenna with High Gain Horn
}

\author{
Guoan Xiong ${ }^{\mathrm{a}}$, Jin $\mathrm{Pan}^{\mathrm{b}}$, Yucheng Jiao ${ }^{\mathrm{c}}$, Zhaonan $\mathrm{Jin}^{\mathrm{d}}$ and Boyuan $\mathrm{Ma}^{\mathrm{e}}$ \\ School of Electronic Engineering, University of Electronic Science and Technology of China, \\ Chengdu 611731, China \\ agustave_xiong@foxmail.com, bpanjin@uestc.edu.cn, ‘610178809@qq.com, \\ d403572765@qq.com, e331953406@qq.com
}

Keywords: slotted waveguide antenna, high gain, cross-polarization, filter.

\begin{abstract}
A low cross-polarization slab waveguide filter for narrow-wall slotted waveguide array antenna with high gain horn is presented. In narrow-wall slotted waveguide array, the horizontal polarization (HP) is the co-polarization, while the vertical polarization (VP) is the cross-polarization. Slotted waveguide array is widely used in radar system, and the high level cross polarization reflects the effect of radar antenna. Physical formulas were presented to give a concise explanation how this structure reduces cross-polarization. The gain of only waveguide slotted array antenna is not high enough for radar system. On this basis, a 40-element low cross-polarization narrow-wall slotted waveguide array antenna with high gain horn in $9.305 \mathrm{GHz}$ was designed and simulated. The antenna has the characteristics of $S L L=-30 \mathrm{~dB}$, cross-polarization lower than $-40 \mathrm{~dB}$ and gain $=32 \mathrm{~dB}$ in main lobe.
\end{abstract}

\section{Introduction}

Because of light weight, low side lobe and convenience to control side lobe, slotted waveguide antenna has been widely used in radar system. Narrow walls or wide walls of waveguide are fabricated in order to interrupt the current distribution. Conduction currents can bypass slots, while displacement currents can traverse slots. For narrow-wall slotted waveguide array antenna, the slant slots with different angles and depths must interrupt the surface currents to radiate TE10 mode wave. The slant slots mainly radiate horizontal polarization wave, while vertical polarization wave is also radiated from them. Cross-polarization in such antenna is not neglectable, which cause additional lobs in azimuth pattern.

Cross-polarization radial component is undesired for narrow-wall slotted waveguide array antenna. Many methods have been presented to decrease cross-polarization. Creating untitled slots in the waveguide can change the fields inside waveguide, which can lower the cross-polarization [1]. [2] presents a slot array waveguide antenna using ridge on its narrow wall and six radiation elements having a frequency of $9 \mathrm{GHz}$. The antenna has the characteristics SLL=-20dB and cross- polarization equivalent to $-60 \mathrm{~dB}$. [3] designed an edge-slotted waveguide antenna array with very low crosspolarization using a pair of shaped irises that flank the slot instead of the aforementioned wires or stripes in the measured result for 16-element slot shows cross-polarization level less than -40dB. Another way to reduce this problem is polarization filter.

Based on recent reports, it seems that most of the works reported on low cross-polarization narrow-wall slotted waveguide array antenna have paid attention to ways to design it. But the analysis method are not reported systematically. In this paper, the narrow wall slotted waveguide array antenna with low cross-polarization slab waveguide filter is designed and simulated by HFSS. The designed array antenna has characteristics cross-polarization lower than $-40 \mathrm{~dB}, \mathrm{SLL}=-30 \mathrm{~dB}$ and gain $=32 \mathrm{~dB}$ in main lobe. 


\section{Antenna Design}

\subsection{Polarization Filter Design.}

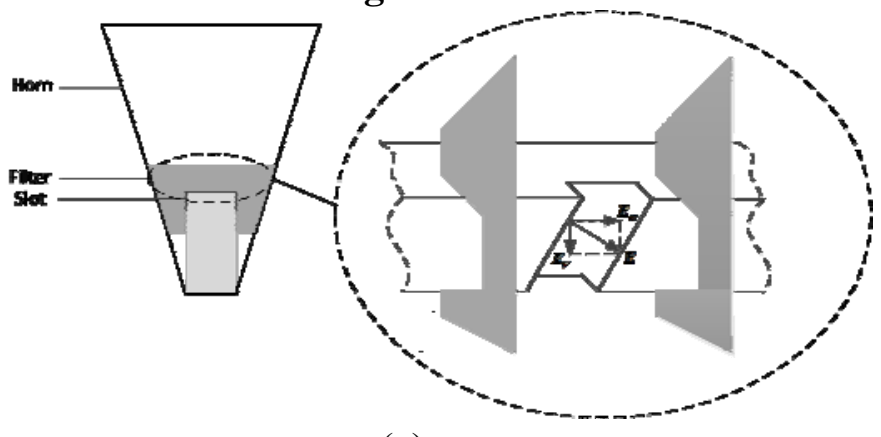

(a)

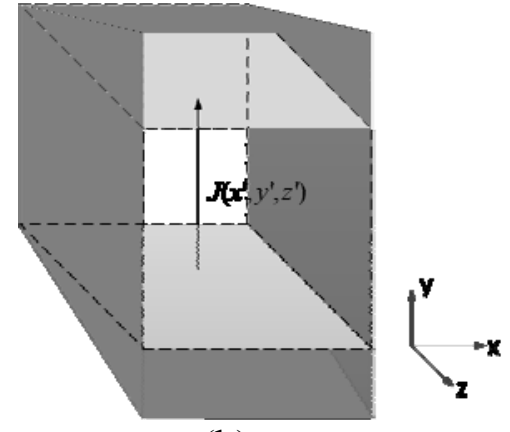

(b)

Fig. 1 (a) Narrow-wall slotted waveguide array antenna with rectangular waveguide polarization filter and its E field distribution (b) Time-harmonic current in semi-unbounded rectangular waveguide system

Because trapezoidal slab waveguide can be regarded as a kind of special gradual rectangular waveguide. Trapezoidal slab waveguide filter can be analyzed by rectangular waveguide mood. The slab waveguide polarization filter is shown in Fig.1(a). Time-harmonic current in semi-unbounded slab waveguide system can be analyzed by in semi-unbounded rectangular waveguide system in Fig.1(b). By means of Babinet's principle [4], many of the problems of slot antennas including slotted waveguide antenna can be reduced to situations solving complementary linear antennas for which solutions have already been obtained. For narrow wall slotted waveguide array antenna, E field is shown in Fig. 1. In Fig. 1, E field can be decomposed into $\boldsymbol{E}_{\boldsymbol{H}}$ and $\boldsymbol{E}_{\boldsymbol{V}}$. By the means of Babinet's principle, $\boldsymbol{E}_{\boldsymbol{H}}$ can be excited by $\boldsymbol{I}_{\boldsymbol{H}}$ in horizontal electric dipole antenna, and $\boldsymbol{E}_{\boldsymbol{V}}$ can be excited by $\boldsymbol{I}_{\boldsymbol{V}}$ in vertical electric dipole antenna.

In a waveguide system, when the operating frequency is less than the cutoff frequency of $\mathrm{TE}_{01}$ wave $\left(f<f_{c}\right), T E 01$ wave mustn't propagated. There is the same E field distribution between TE01 wave in a rectangular waveguide system and vertical electric dipole antenna. For the purpose of reducing $\boldsymbol{E}_{V}$ (cross-polarization), the condition as bellow must be fulfilled to ensure the falloff of TE 01 mood,

$$
b<\lambda / 2
$$

where: $b$-length of narrow wall of waveguide filter; $\lambda$-length of electromagnetic in a free space

To ensure the propagation of $\mathrm{TE}_{10}$ mood (co-polarization), the condition as bellow must be fulfilled

$$
a>\lambda / 2
$$

where: a-length of broad wall of waveguide filter; $\lambda$-length of electromagnetic in a free space

In order to ensure the cross-polarization field in this kind of system, slots can be regarded as the excitation source for above semi-unbounded rectangular waveguide system in Fig.1(b). E field excited by y direction current $\boldsymbol{J}\left(\boldsymbol{r}^{\prime}\right)$ in $\left(\mathrm{x}^{\prime}, \mathrm{y}^{\prime}, \mathrm{z}^{\prime}\right)$ can be presented by electric dyadic Green function of the first kind as follows

$$
\boldsymbol{E}(\boldsymbol{r})=-j \omega \mu \int_{V} \overline{\overline{G_{1}^{e}}}\left(\boldsymbol{r}, \boldsymbol{r}^{\prime}\right) \cdot \boldsymbol{J}\left(\boldsymbol{r}^{\prime}\right) d V^{\prime}
$$

By dyadic Green functions in electromagnetic theory, we obtain

$$
\begin{aligned}
& \nabla \times \nabla \times \overline{\overline{G_{1}^{e}}}\left(\boldsymbol{r}, \boldsymbol{r}^{\prime}\right)-k^{2} \overline{\overline{G_{1}^{e}}}\left(\boldsymbol{r}, \boldsymbol{r}^{\prime}\right)=-\boldsymbol{e}_{y} \boldsymbol{e}_{y} \delta\left(r-r^{\prime}\right) \\
& G\left(\boldsymbol{r}, \boldsymbol{r}^{\prime}\right)=\frac{2}{a b} \sum_{m=1}^{\infty} \sum_{n=0}^{\infty} \frac{2-\delta_{n 0}}{\gamma_{m n}} \sin \frac{n \pi x}{a} \sin \frac{n \pi x^{\prime}}{a} \cos \frac{m \pi y}{b} \cos \frac{m \pi y^{\prime}}{b} \cdot \begin{cases}e^{-\gamma_{m n} z^{\prime}} \operatorname{sh} \gamma_{m n} z & z \leq z^{\prime} \\
e^{-\gamma_{m n} z} \operatorname{sh} \gamma_{m n} z^{\prime} & z \geq z^{\prime}\end{cases} \\
& \overline{\overline{G_{1}^{e}}}\left(\boldsymbol{r}, \boldsymbol{r}^{\prime}\right)=\boldsymbol{e}_{x} \boldsymbol{e}_{y} \frac{1}{k^{2}} \frac{\partial^{2} G\left(\boldsymbol{r}, \boldsymbol{r}^{\prime}\right)}{\partial x \partial y}+\boldsymbol{e}_{y} \boldsymbol{e}_{y}\left[G\left(\boldsymbol{r}, \boldsymbol{r}^{\prime}\right)+\frac{1}{k^{2}} \frac{\partial^{2} G\left(\boldsymbol{r}, \boldsymbol{r}^{\prime}\right)}{\partial y^{2}}\right]+\boldsymbol{e}_{z} \boldsymbol{e}_{y} \frac{\partial^{2} G\left(\boldsymbol{r}, \boldsymbol{r}^{\prime}\right)}{\partial z \partial y}
\end{aligned}
$$


where: $\gamma_{m n}^{2}=\left(\frac{m \pi}{a}\right)^{2}+\left(\frac{n \pi}{b}\right)^{2}-k^{2} ; \delta_{n 0}= \begin{cases}1 & n=0 \\ 0 & \text { else }\end{cases}$

\subsection{Horn Design.}

A horn antenna may be regarded as a flared-out waveguide [5]. The function of the horn is to produce a uniform phase with a larger aperture than that of the waveguide and hence greater directivity. Assuming that the rectangular waveguide is energized with a $\mathrm{TE}_{10}$ mode wave electric field, the horn in Fig. 2(a) flared out in a plane perpendicular to E. This is the plane of the magnetic field $\mathrm{H}$. Hence, this type of horn is called a sectoral horn flared in the $\mathrm{H}$ plane or an $\mathrm{H}$-plane sectoral horn. Optimum horn dimensions can be got in (7)(8).

$$
\begin{aligned}
& \delta=\frac{L}{\cos (\theta / 2)}-L \\
& L=\frac{\delta \cos (\theta / 2)}{1-\cos (\theta / 2)}
\end{aligned}
$$

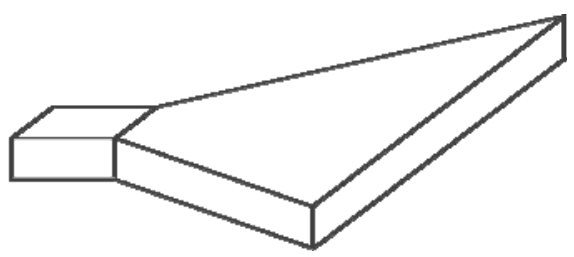

(a)

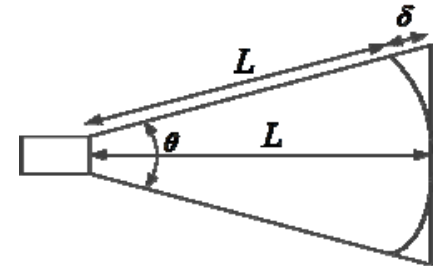

(b)

Fig. 2 (a) H-plane sectoral horn (b) Design parameters of H-plane sectoral horn

\subsection{Array antenna Design.}

Fig. 3 shows the structure of 40-element slotted waveguide array antenna with slab waveguide cross-polarization filter. $\mathrm{BJ} 100$ waveguide $(\mathrm{a}=22.86 \mathrm{~mm}, \mathrm{~b}=10.16 \mathrm{~mm}, \mathrm{t}=1.27 \mathrm{~mm})$ is chosen to be slotted. The filter is made up of FR4 layer with copper of $0.018 \mu \mathrm{m}$ on it. Table 1 shows the structure parameters. By Taylor synthesis method, the amplitude of each slot in 40 elements of SLL $=30 \mathrm{~dB}$ can be get. The equivalent normalized conductivity distribution is also calculated by [6] as Fig. 4(a). The equivalent normalized conductivity distribution is the maximum at an angle among different depths. Using (9), the relationships among angle, equivalent normalized conductivity g and slot depth can be simulated by HFSS. The correspondence among angle, equivalent normalized conductivity $g$ and slot depth can be shown in Fig. 4(b). Using Fig. 4(a) and 4(b), the angle and depth of each slot can be sure.

$$
g=e^{-2 \alpha d}\left(\frac{S_{12}^{2}}{1-S_{11}^{2}}\right)^{-\frac{1}{N}}-1
$$

where: $\alpha$-waveguide loss per unit length; $N$-number of slots

Table 1 Structure Parameters

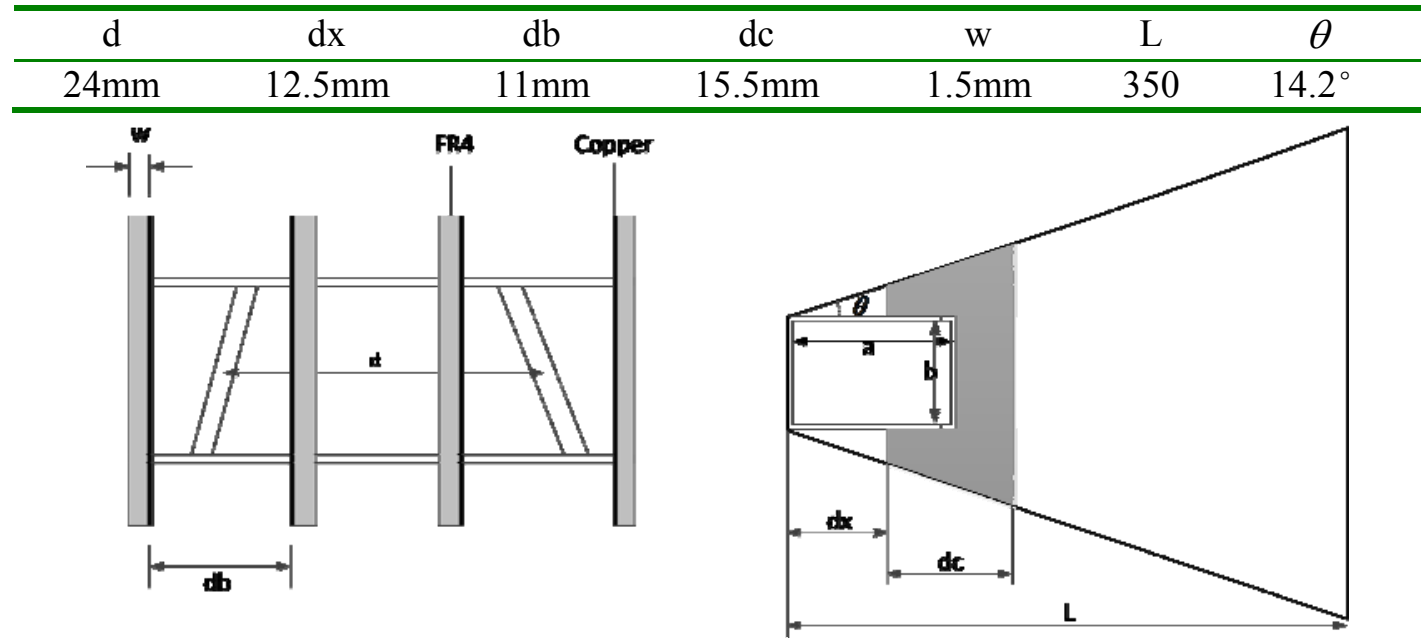

Fig. 3 Slotted waveguide array antenna with slab filter and horn 
Fig. 5 shows the simulated radiation pattern in horizontal plane in 40-element slotted waveguide antenna with cross-polarization filter in $9.305 \mathrm{GHz}$. The simulated maximum side lobe level is $-30 \mathrm{~dB}$ with cross-polarization level of $-40 \mathrm{~dB}$ below the main lobe in the band.

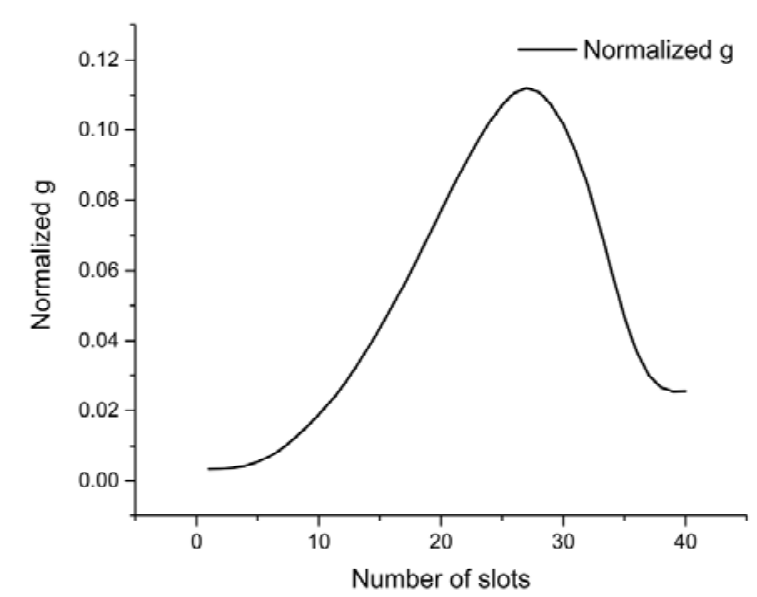

(a)

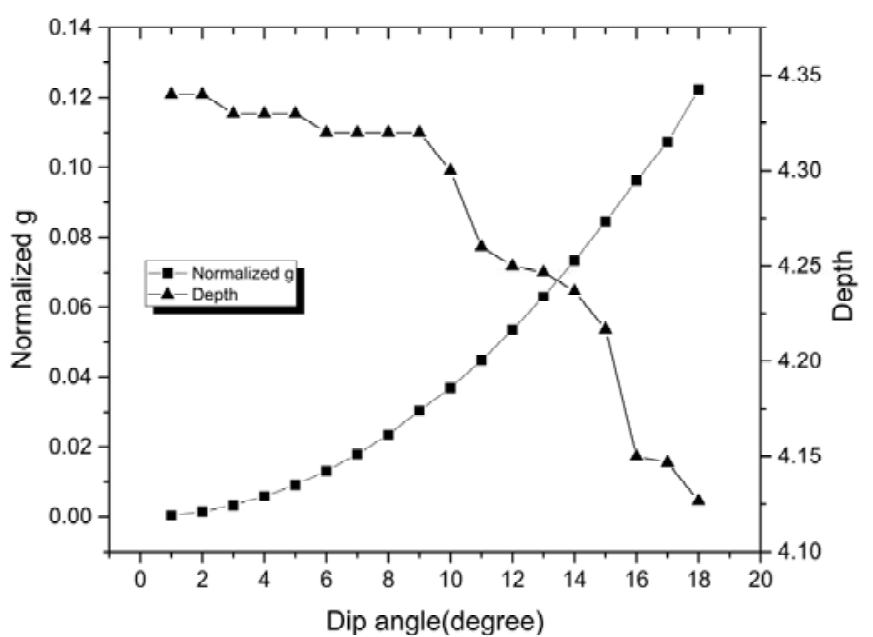

(b)

Fig. 4 (a) Equivalent normalized conductivity distribution (b) Correspondence among angle, equivalent normalized conductivity g and slot depth

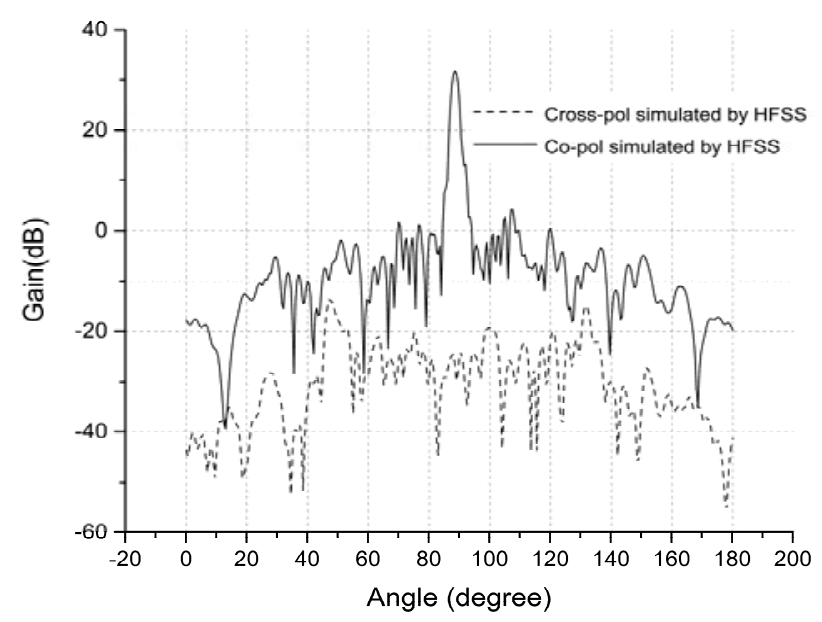

Fig. 5 Simulated radiation pattern in horizontal plane in $9.305 \mathrm{GHz}$

\section{Summary}

In this paper the principle of decreasing cross-polarization by slab cross-polarization filter and high gain by horn antenna is reported. On this basis, 40-element narrow wall slotted waveguide array antenna with high gain horn in $9.305 \mathrm{GHz}$ with this filter is designed and simulated by HFSS. The level of cross-polarization lobes below the main lobe is lower than $-40 \mathrm{~dB}$ with the maximum side lobe level of $-30 \mathrm{~dB}$ and $32 \mathrm{~dB}$ gain in main lobe, allowing this antenna adapt to radar system.

\section{References}

[1]. W. Wang, J. Jin, J. G. Lu, et al. "Waveguide slotted array antenna with broadband, dual-polarization and low cross-polarization for X-band SAR applications." IEEE International Radar Conference. Arlington, Virginia, USA, 9-12, May, 2005, p. 653-656.

[2]. A. R. Mallahzadeh, S. M. A. Nezhad. "Cross-Polarization and Size Reduction of Slotted Waveguide Array Antenna by Angled Ridges." European Conference on Antennas and Propagation. Prague, Czech Republic, 26-30, March, 2012, p.1-5. 
[3]. W. Wang, S. S. Zhong, J. Jian, et al. "An untilted EDGE-slotted waveguide antenna array with very low cross-polarization." Microwave \& Optical Technology Letters. Vol. 44(2005), p. 91-93.

[4]. H. G. Booker, "Slot aerials and their relation to complementary wire aerials (Babinet's principle)." Electrical Engineers - Part IIIA: Radiolocation. Vol. 93 (1946) No. 4, p. 620-626.

[5]. J. D. Kraus, R. J. Marhefka. "Antennas for all applications." Publishing House of Electronics Industry, 2008, p. 330-333.

[6]. A. F. Stevenson, "Theory of Slots in Rectangular Wave - Guides." Journal of Applied Physics. Vol. 19 (1948) No. 1, p. 24-38. 